\title{
The method of forming probing signals of georadars on the basis of direct digital synthesizers for geodynamic monitoring
}

\author{
$A V$ Grecheneva $^{1}, N V$ Dorofeev $^{1}, O R$ Kuzichkin $^{1, *}, D I$ Surzhik $^{2}$, and $R V$ Romanov $^{2}$ \\ ${ }^{1}$ Belgorod State University, 85 Pobedy St, Belgorod, 308015, Russia \\ ${ }^{2}$ Vladimir State University, 87 Gor'kogo St, Vladimir, 600000, Russia
}

\begin{abstract}
The features of the formation of probing signals based on direct digital synthesizers for geodynamic monitoring of medias using georadar probing are considered in the article. The basic requirements for the formers of probing signals and the shortcomings of known technical solutions are shown. To eliminate them, as a former of probing signals of georadars was suggested to use direct digital synthesizers with improved spectral characteristics, achieved by introducing circuit of an automatic compensation of phase distortion with a control device in the form of a phase-locked loop. With this construction, the device contains a combination of autocompensation and filter methods of improving the spectral characteristics of the output signal of the synthesizer. To analyze the effectiveness of the proposed solutions, were obtained the necessary mathematical relationships describing the functioning of the device under research and implemented its schematic simulation, which showed the possibility of improving the spectral characteristics of the proposed former of probing signals by $10-15 \mathrm{~dB}$.
\end{abstract}

\section{Introduction}

One of the actively developing methods of geodynamic monitoring is high-frequency georadar probing [1-3], based on the propagation of radio waves with frequencies above $100 \mathrm{kHz}$ and researching their reflection from the borders of layers of the probed medium having different dielectric permittivities. Such boundaries are, for example, contacts between dry and moisture saturated soils, between rocks of different lithologic composition, between rock and artificial construction material, between frozen and thawed soils, etc.

At present, georadars implementing in practice the considered method of georadar probing are widely used to research objects lying at a relatively shallow depth $(0.2-40 \mathrm{~m})$ $[1,2]$. Depending on the task to be solved when probing, the permissible resolving power of the research and the associated probing frequency are determined, the range of which can change, as a rule, from 0.1 to $2000 \mathrm{MHz}$. In this case, an increase in the probing frequency leads to an improvement in the resolving power of the method, which, however, is accompanied by an increase in the attenuation of the propagated radio wave in the medium

\footnotetext{
* Corresponding author: 1155464@bsu.edu.ru
} 
and, accordingly, leads to a decrease of the probing depth and vice versa. For example, with a probing frequency of $500 \mathrm{MHz}$, the resolution of the method is $0.5 \mathrm{~m}$ with a maximum depth of about $10 \mathrm{~m}$, and at a probing frequency of $40 \mathrm{MHz}$ the resolution is $4 \mathrm{~m}$ with a maximum depth of $30 \mathrm{~m}$.

One of the promising and actively developing in recent years variants of georadars is a multi-frequency ground penetrating radar with a synthesized aperture [4]. An important independent task in its practical implementation is the choice of the device of forming probing signals. These devices should ensure the coherence of a discrete set of generated frequencies in a given frequency spectrum range; precise control of their amplitudes, frequencies and phases; high stability and speed under the conditions of various kinds of natural and technogenic interferences. The main drawbacks of the known methods for forming probing signals are undesirable changes in the phase of the generated signals when tuning from one frequency to another and the complexity of providing phase synchronization.

\section{The use of direct digital synthesizers with improved spectral characteristics}

The most significant shortcomings of the known methods for forming probing signals can be eliminated by the method of direct digital frequency synthesis, realized with the help of direct digital synthesizers (DDS) [5-9]. Its most important advantages are precision of synthesized signals, extremely high resolution in frequency (up to thousandths of $\mathrm{Hz}$ ) and speed.

The main disadvantage of direct digital synthesis of frequencies at the moment remains insufficient spectral purity of synthesized signals. It is connected with the presence of phase distortions of the output signal [10] of the DDS, which lead to the formation of phase noise and a lot of discrete parasitic spectral components (PSCs) in the frequency domain. Sources of PSCs of phase origin in the spectrum of the output signal of the DDS are destabilizing factors and the effect of truncation of the phase code, sources of phase noises are the quantization effect and the device's own noises.

To improve the spectral characteristics of the DDS and various options for constructing of formers of probing signals on their basis, it is proposed to use the method of automatic compensation of phase distortion (ACPD) [11,12]. The authors developed an original algorithm for isolating and compensating for phase distortions of the DDS, as well as several versions of the structural circuits of the formers of probing signals based on it.

Based on the specifics of the method of georadar probing, the most effective option of the realization of the former of the probing signals is the block diagram of the device shown in figure 1. It is a serial connection of a clock generator (CG), a DDS with circuit of autocompensation of phase distortions and a phase locked loop (PLL). The following designations are adopted on the block diagram: LPF DDS - output low-pass filter of the DDS, PD - phase detector, LPF - low-pass filter, SD - subtractor device, VCO - voltage controlled oscillator, FD - frequency divider (with variable or fixed division coefficient), RT - the reference tract of the ACPD, IT - the information tract of the ACPD, CT - the control tract of the ACPD.

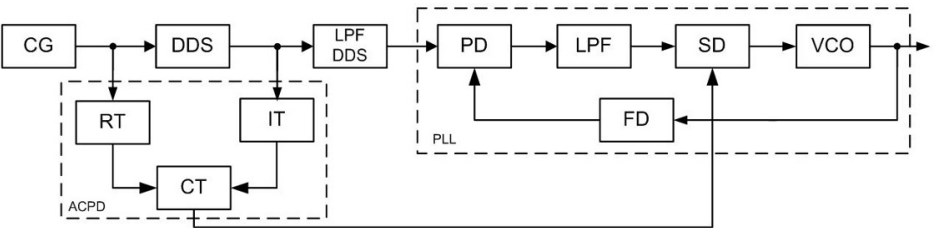

Fig. 1. Block diagram of the former of the probing signals with the ACPD of the DDS. 
A similar combination of the serial connection of the DDS with the autocompensation circuit and the PLL represents a hybrid frequency synthesizer in which the DDS is used as a fractional divider of the output frequency of the clock generator and the reference oscillator for the PLL. With this construction of the former of the probing signals, the high resolution of the device in frequency is provided by the DDS, and the output frequency range is either due to the DDS (in the absence of a frequency divider in the feedback loop of the PLL) or the frequency multiplier based on the PLL.

Improvement of the spectral characteristics of the former of the probing signals is achieved by introducing into the scheme of the device a circuit of autocompensation of the phase distortions of the DDS formed by the reference, information and control tracts. The reference tract is a $\mathrm{T}$ flip-flop, the information tract is a serial connection of a differentiating circuit, an amplifier, a full-wave rectifier and a $\mathrm{T}$ flip-flop, and the control tract is a series connection of a phase detector, a low-pass filter, and a amplifier.

The processing of the input and output signals of the DDS in the reference and information tracts makes it possible to align their shapes and amplitudes with the preservation of phase shifts proportional to the phase distortions isolated in the control tract $[11,12]$. Compensation of this phase distortions of the output signal of the DDS is carried out by changing the modulating voltage of the voltage controlled oscillator of the PLL of the former of the probing signals by subtracting the compensating signal (in the SD) formed in the control tract of the ACPD. In this case, the PLL simultaneously performs the function and control device of the autocompensator and the high-efficiency low-pass filter for the input phase distortions of the DDS and the clock generator. As a result, the considered device contains in its composition a combination of two methods for improving the spectral characteristics - autocompensating and filter methods.

To derive the basic mathematical relationships describing the functioning of the former of the probing signals under the action of phase deviations of the output signal of the DDS, its functional diagram is compiled - figure 2. In general, the device contains three blocks with nonlinear characteristics of the detectors (of the PLL and autocompensator) and the voltage-controlled oscillator, which, under the assumption about of small values of phase deviations, can be linearized by replacing the corresponding steepnesses. The following symbols are used in the scheme: $\Delta \varphi_{\mathrm{CG}}$ - the deviation of the phase of the output signal of the clock generator; $\Delta \varepsilon$ - the impact on the DDS in the form of a phase deviation; $\Delta \varphi_{\mathrm{VCO}}-$ the deviation of the phase of the output signal of the voltage controlled oscillator; $K_{\mathrm{DDS}}$ - the transfer coefficient of the DDS; $K_{\varepsilon}$ - the transmission coefficient of phase deviations of the $\mathrm{DDS} ; K_{\mathrm{PD}}$ - the steepness of the detector characteristic of PD of the PLL; $M(p)$ - the transfer factor of the low-pass filter of the PLL; $p$ - the Laplace operator; $K_{\mathrm{VCO}}$ - steepness of the modulation characteristic of the voltage controlled oscillator; $N$ - the division coefficient of the frequency divider; $K_{\mathrm{PDA}}$ - the steepness of the detector characteristic of the phase detector of the ACPD; $n_{\mathrm{A}}$ - the gain of amplifier in the control tract of the ACPD; $M_{\mathrm{A}}(p)$ transfer factor of the low-pass filter of the ACPD.

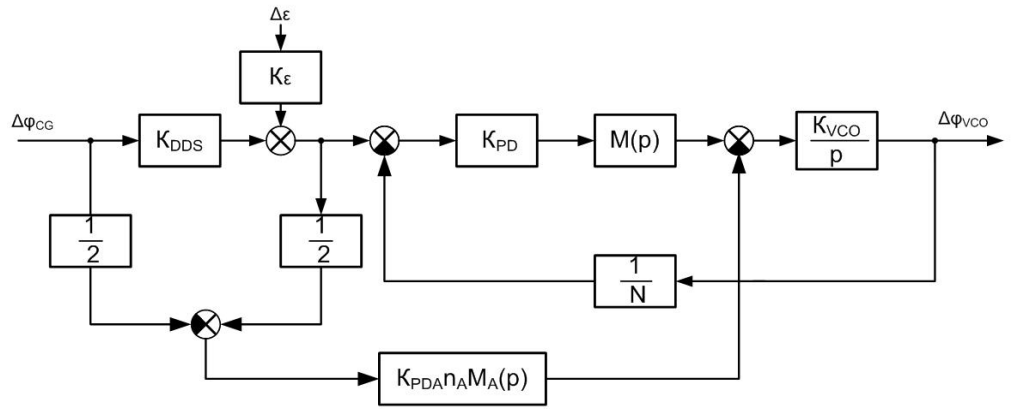

Fig. 2. Functional diagram of the former of probing signals with the ACPD of the DDS. 
According to figure 2, the linearized differential equation describing the functioning of the former of the probing signals for the phase deviations of the output signal of the DDS is determined by

$$
\Delta \varphi_{V C O}\left(1+\frac{K_{V C O} K_{P D} M(p)}{N p}\right)=\Delta \varepsilon \frac{K_{V C O} \alpha}{p},
$$

where $\alpha=K_{V C O} K_{P D} K_{\varepsilon} M(p)-\frac{1}{2} K_{V C O} K_{P D A} K_{\varepsilon} n_{A} M_{A}(p)$.

The equation obtained completely describes the processes occurring in the former of the probing signals for any phase deviations of the output signal of the DDS with arbitrary filter configurations and device blocks parameters.

Using the differential equation (1), the transfer function of the device is obtained, which describes the transformation of undesirable deviations of the phase of the output signal of the DDS as they pass to the output of the former of the probing signals.

$$
H_{\Delta \varepsilon \Delta \varphi_{V C O}}=\frac{\Delta \varphi_{V C O}}{\Delta \varepsilon}=\frac{N_{\varepsilon} \frac{M(p)}{p}-N_{A} \frac{M_{A}(p)}{p}}{1+\frac{M(p)}{T_{P L L} p}},
$$

where $T_{P L L}=\frac{N}{K_{V C O} K_{P D}}$ is the own PLL time constant, $N_{\varepsilon}=K_{V C O} K_{P D} K_{\varepsilon}$ - the transfer factor of the phase deviation, $N_{A}=\frac{1}{2} K_{V C O} K_{P D A} K_{\varepsilon} n_{A}$ - the ACPD regulation factor.

The analysis of this expression allows to conclude that in order to ensure complete compensation of phase distortions of the DDS when using regulation by the perturbation, it is necessary to ensure equality the zero of the numerator of the transfer function of the device (2), whose solution with respect to the parameters of the autocompensation circuit gives the following conditions for complete compensation of the phase distortions of the DDS

$$
n_{A}=2 \frac{K_{P D}}{K_{P D A}}, M_{A}(p)=M(p) \text {. }
$$

\section{Schematic modeling of the former of the probing signals with improved spectral characteristics}

To confirm the effectiveness of the proposed method for improving the spectral characteristics of the DDS and the formers of the probing signals on their basis, a schematic simulation of the device under investigation in the program for modeling the electric and electronic circuits Micro-Cap was conducted.

In this model, the DDS is implemented using specially created macros. The first describes the functioning of the synthesizer, taking into account the discharges of its phase accumulator (PA), a read-only memory (ROM), a digital-to-analog converter (DAC) and the main sources of phase distortions: the quantization effect, truncation of the phase code when it is addressed from PA to ROM and impact of destabilizing factors. The second macro of the DDS model is designed to reproduce the real nonlinearity of the DAC conversion.

The loop of the digital PLL (without a frequency divider in the feedback loop) of the model of the former of the probing signals contains a phase detector on the on the logical 
element XOR, a first-order low-pass filter and a voltage-controlled oscillator. As models of clock generator of the former, the subtracting device and the structural blocks of the autocompensation circuit of the phase distortions of the DDS the models of idealized blocks are used.

In figures 3 and 4 show the spectrums of the output signals of the DAC of the DDS and the former of the probing signals (in the presence and absence of the autocompensation circuit of the phase distortions of the DDS) in the frequency band of the main output signal of the synthesizer 7.2 MHz. As parameters of the DDS, values close to those of the real AD9854 synthesizer manufactured by Analog Devices were used: the clock frequency 30 $\mathrm{MHz}$, phase accumulator with 32-bit capacity, ROM with 16-bit capacity and DAC with 12-bit capacity.

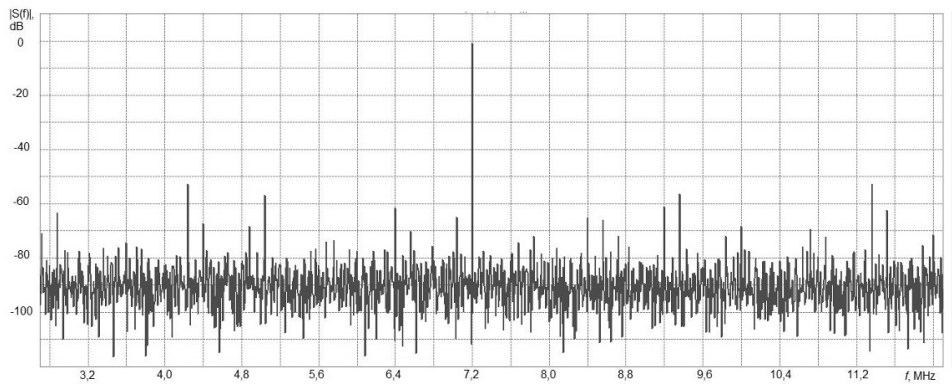

Fig. 3. Spectrum of output signal of the DAC of DDS.

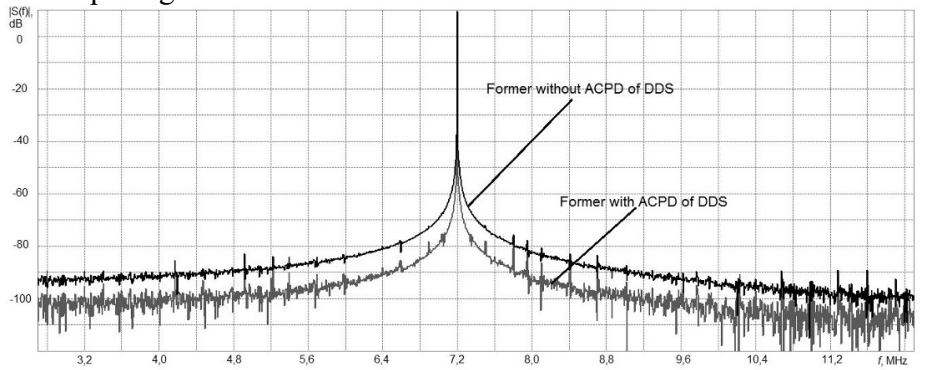

Fig. 4. Spectrums of the output signals of the former of the probing signals.

From the presented spectral characteristics it is seen that for the given parameters of the former of the probing signals, the spectral characteristics of the device are improved by 8 $\mathrm{dB}$ due to the introduction the autocompensation circuit of phase distortions of the DDS into the circuit of device. Based on the results of the simulation, it was revealed that, depending on the accuracy of setting the autocompensator blocks (in accordance with conditions (3)), this parameter can reach 10-15 dB. In addition, it is established that the passing output signal of the DAC of the synthesizer through the output filter of the DDS and the PLL is accompanied by the expansion of the spectral line of the harmonic of the main tone in the band of detunings from the carrier frequency by $0-600 \mathrm{kHz}$.

\section{Conclusion}

The use of georadar probing in the implementation of geodynamic monitoring makes it possible to effectively solve both geological, hydrogeological and engineering-geological problems, as well as tasks for locating objects, inspecting engineering structures, etc., and the application DDSs with improved spectral characteristics for forming probing signals of georadars allows eliminating the main disadvantages of known methods of formation, increase the geodynamic sensitivity of georadars and eliminate undesirable phase changes when the tuning of the probing frequencies. The conducted schematic simulation confirms 
the operability of the proposed devices and the effectiveness of applying the method of autocompensation to reduce phase distortions of the output signal of the DDS.

As part of the further development of the theme of this article, it is planned to develop a structural scheme of a multifrequency georadar with a synthesized aperture and using the proposed device as a former of probing signals, and to conduct its theoretical and experimental researches.

\section{Acknowledgments}

The work was carried out as part of the research on the project, support by the Ministry of Education and Science of the Russian Federation No. 5.3606.2017/PCH.

\section{References}

1. Khmelevskoy V K 1989 Handbook of Exploration Geophysics vol 2, ed V K Khmelevskoy and V M Bondarenko (Moscow: Nedra) p 438

2. Fedynsky V V 1964 Exploration geophysics (Moscow: Nedra) p 677

3. Kuzichkin O, Mikhaleva E, Dorofeev N and Romanov R 2017 IEEE 9th Int. Conf. on IDAACS 21-23 Sept. vol 1 (Bucharest: IEEE) pp 227 - 230

4. Finkelstein M I Mendelson V L and Kutev VA 1977 Radiolocation of layered earth cover. Ed M I Finkelstein. (Moscow: Sov. Radio) p 176

5. Vankka J and Halonen K 2000 Direct Digital Synthesizers: Theory, Design and Applications (Helsinki: University of Technology) p 208

6. Kroupa V F 2003 Phase Lock Loops and Frequency Synthesis (John Wiley \& Sons, Ltd) p 320

7. Goldberg B 1999 Digital Frequency Synthesis Demystified DDS and Fractional-N PLLs (Eagle Rock VA: LLH Technology Publishing) p 355

8. Ridiko L I 2001 Components and technologies vol 17 (St. Petersburg: Publisher Fainstreet) pp50-56

9. Kester W 2007 Analog-to-digital conversion (Moscow: Technosphere) p 1016

10. Peng L 2009 Jitter Electronic components vol 10 pp 81-85

11. Surzhik D I, Kurilov I A, Kuzichkin O R, Vasilyev G S and Kharchuk S M 2015 Int. Conf. on Control and Communications (Omsk: IEEE) pp 1-7

12. Vasilyev G S, Kuzichkin O R, Kurilov I A and Surzhik D I 2016 International Int. Conf. on Control and Communications (Moscow: IEEE) pp 1-6 